\title{
Trifecta outcomes of modified robot-assisted simple enucleation and standard robot-assisted partial nephrectomy for treating clinical T1b renal cell carcinoma
}

\author{
Xiaozhi Zhao", Qun Lu\#, Changwei Ji, Guangxiang Liu, Xuefeng Qiu, Shiwei Zhang, Xiaogong Li, \\ Gutian Zhang, Hongqian Guo
}

Department of Urology, Drum Tower Hospital, Medical School of Nanjing University, Institute of Urology, Nanjing University, Nanjing, China

Contributions: (I) Conception and design: X Zhao, Q Lu, H Guo; (II) Administrative support: H Guo, G Zhang; (III) Provision of study materials or patients: C Ji, G Liu; (IV) Collection and assembly of data: Q Lu, X Qiu; (V) Data analysis and interpretation: X Zhao, S Zhang, X Li; (VI) Manuscript writing: All authors; (VII) Final approval of manuscript: All authors.

\#These authors contributed equally to this work.

Correspondence to: Hongqian Guo. Department of Urology, Drum Tower Hospital, Medical School of Nanjing University, Institute of Urology, Nanjing University, 321 Zhongshan Rd., Nanjing 210008, China. Email: dr.ghq@nju.edu.cn.

\begin{abstract}
Background: To compare perioperative outcomes and Trifecta achievement of modified robot-assisted simple enucleation (MRASE) with robot-assisted partial nephrectomy (RAPN) for treating clinical T1b renal tumors.

Methods: We analyzed 203 patients who underwent MRASE or RAPN for clinical T1b renal tumors at our institution from September 2014 to June 2018. The two groups were compared regarding perioperative outcome variables. Trifecta was defined as no perioperative complications, negative surgical margin, and ischemia time $\leq 25$ minutes.

Results: In all, 139 patients underwent MRASE and 64 underwent RAPN respectively. Patients in the MRASE group had shorter operative time (197.7 vs. $215.6 \mathrm{~min}, \mathrm{P}=0.039)$ and warm ischemic time (21.2 vs. $24.1 \mathrm{~min}, \mathrm{P}=0.004)$ in comparison to the RAPN group. The groups were comparable in estimated blood loss (230.5 vs. $269.8 \mathrm{~mL}, \mathrm{P}=0.259)$. Tumor bed suturing was performed with a significantly lower frequency in the MRASE group than in the RAPN group ( $11.5 \%$ vs. $90.6 \%, \mathrm{P}<0.01)$. The incidence of perioperative complications was similar. The rate of positive surgical margins was similar in both groups $(2.2 \% \mathrm{vs}$. $6.3 \%, \mathrm{P}=0.284)$. Trifecta was achieved in $61.2 \%$ and $42.2 \%$ of MRASE and RAPN patients $(\mathrm{P}=0.012)$. On multivariable analysis, the type of procedure, RENAL score, estimated blood loss, and operative time were positive factors for the achievement of Trifecta.

Conclusions: In this series MRASE was superior to RAPN with regard to the achievement of Trifecta in treating T1b renal tumors. Besides, MRASE had better outcomes for shorter operative time, shorter warm ischemic time, and less need for tumor bed suturing as compared with RAPN.
\end{abstract}

Keywords: Renal cell carcinoma (RCC); robot-assisted; partial nephrectomy; simple enucleation; Trifecta

Submitted Aug 08, 2020. Accepted for publication Jan 05, 2021.

doi: $10.21037 / \mathrm{tau}-20-1153$

View this article at: http://dx.doi.org/10.21037/tau-20-1153

\section{Introduction}

Renal cell carcinoma (RCC) currently represents $2-3 \%$ of all adult malignant tumors $(1,2)$. Partial nephrectomy $(\mathrm{PN})$ is considered the standard surgical procedure that excides the tumor with a visible margin of normal renal parenchyma (3). PN has now become the standard treatment for patients with clinical T1a renal tumors, and it is also applied for T1b renal tumors whenever technically 
feasible $(4,5)$. In the last decades, it is debate what is the suitable width of normal tissue excised with the tumor to ensure negative margins. Some studies have revealed that the width of resected normal parenchyma can be reduced to only a few millimeters without influencing the oncologic safety $(6,7)$. In this context, several authors have proposed the application of the simple enucleation (SE) technique, in which the tumor is removed by the blunt excision without a visible rim of normal parenchyma and maximal amount of renal parenchyma can be preserved $(8,9)$.

Robot-assisted partial nephrectomy (RAPN) was first proposed by Gettman in 2004 (10). This surgical technique is considered as a feasible and effective alternative to open and laparoscopic PN for treating renal tumors $(11,12)$. With the advantages such as fully articulating instruments and magnified 3D imaging, the da Vinci Surgical System can overcome the technical difficulties and shorten the learning curve of minimally invasive PN (13). Robotassisted SE (RASE), which can reserve maximal renal parenchyma theoretically without effecting the safety of oncology (14), may be a substitute for RAPN. However, few data comparing RASE and RAPN for T1b renal tumors have been reported.

Here, we compared the achievement of Trifecta and the perioperative results between modified robot-assisted simple enucleation (MRASE) and RAPN for treating clinical T1b renal tumors based on our large institutional experience. We present the following article in accordance with the STROBE reporting checklist (available at http:// dx.doi.org/10.21037/tau-20-1153).

\section{Methods}

\section{Patients}

From September 2014 to June 2018, we gathered the perioperative data from 218 consecutive patients treated with MRASE or RAPN for clinical T1b renal tumors. Nine patients without imaging for nephrometry scoring were excluded from the study. Patients with multifocal tumors $(\mathrm{n}=1)$ and solitary kidneys $(\mathrm{n}=5)$ were also excluded. A total of 203 patients were included in the study, of whom 139 patients underwent MRASE and 64 underwent RAPN. All the operations were performed by Doctor Hongqian Guo. The study was conducted in accordance with the Declaration of Helsinki (as revised in 2013). The study was approved by institutional ethics committee of Nanjing Drum Tower Hospital (2019-153-02) and informed consent was taken from all the patients.

Clinical records of patient demographics, perioperative outcomes and pathological findings were assessed. Physical condition was classified by the Eastern Cooperative Oncology Group (ECOG) criteria (15). The tumor presentation mode was classified according to the Patard classification (16). The RENAL nephrometry scoring system was applied to assess the tumor complexity by a radiologist and a urologist (17). Preoperative and postoperative complications were classified according to the modified Clavien-Dindo system (18). The modification of diet in renal disease formula was used to calculate the estimated glomerular filtration rate (eGFR) (19). For the last eGFR measurement, the eGFR at least 1 year after surgery was used. Trifecta was defined as a combination of negative surgical margin, zero perioperative complications, and ischemia time $\leq 25 \mathrm{~min}(20)$.

\section{Surgical technique}

The surgical choice of RAPN or MRASE was determined mainly based on the tumor characteristics prior to surgery. Tumor enucleation was implemented according to imageological examination and intraoperative evaluation. The tumors with intact and distinct capsule displayed on ultrasonic contrast or radiography were mostly enucleated. Tumors with distinct borders were mainly characterized by the regular shape and clear boundaries with normal renal parenchyma.

The surgical techniques and placement of trocar in RAPN were reported in previous studies (21). We have described some important variations of SE technique in our published studies which was mentioned in the surgical procedure part below $(8,14)$. The transperitoneal approach was generally applied, and the patients were positioned in flank position. Operations were performed using the Si da Vinci surgical system (Intuitive Surgical, Sunnyvale, USA) docked at the back of the patients. A three-arm arrangement and 30-degree downward-angled optics were applied in most situations. Monopolar scissors on the right arm and Fenestrated/Maryland graspers on the left arm were commonly used.

The renal pedicle was carefully isolated in advance. The kidney was completely isolated from the perirenal fat to exclude satellite lesions and determine tumor limits. For MRASE, the tumor is enucleated initially close to the tumor edge without warm ischemia. When the tumor capsule was reached or when bleeding interfered with the operative view, 
the renal artery was clamped with vascular clamps. The surgeon enucleated the tumor by blunt dissection following the pseudocapsule, without visible normal parenchyma. Once the renal capsule was incised and the natural plane was reached, the tumor could be easily enucleated. After the tumor was removed, the tumor bed was checked to exclude tumor infiltration. Hemostasis was achieved with bipolar coagulation, and incidental opened calices was repaired by a single suture. The cortical defect was usually closed with horizontal and interrupted sutures using Hem-o-lok clips placed at the beginning and end of the suture on the renal capsule. It was defined as the single-layer renorrhaphy technique. At last, the vascular clamp was removed, and additional sutures were performed if necessary. In contrast, RAPN included the sharp excision of the tumor and a thin rim of normal tissue around the tumor. The tumor bed and the cortical defect were both closed with a running suture.

\section{Statistical analysis}

Categorical variables were reported as proportions, whereas continuous variables were reported as mean \pm standard deviation (SD). Student's t-test was performed to compare continuous variables, and chi-square test or Fisher's exact test to compare categorical variables. Univariable and multivariable logistic regression models were used to evaluate the factors predicting Trifecta achievement. All statistical analyses were two-sided with statistically significant $\mathrm{P}$ value $<0.05$. All the analyses were performed with SPSS 17.0 (IBM Corp., Armonk, USA).

\section{Results}

Clinical characteristics and perioperative data were summarized in Table 1. Overall, 139 patients underwent MRASE and 64 underwent RAPN. The two groups were similar in terms of mean age, body mass index (BMI), gender, ECOG score, tumor side, symptoms at diagnosis, clinical tumor size, RENAL score, and preoperative eGFR.

In the MRASE group, the patients had shorter operative time (197.7 vs. $215.6 \mathrm{~min}, \mathrm{P}=0.039$ ) and warm ischemic time (WIT) (21.2 vs. $24.1 \mathrm{~min}, \mathrm{P}=0.004$ ) in comparison with the RAPN group. Tumor bed suturing was performed with a significantly lower frequency in the MRASE group than in the RAPN group ( $11.5 \%$ vs. $90.6 \%, \mathrm{P}<0.01)$. The estimated blood loss was similar in the two groups (230.5 vs. $269.8 \mathrm{~mL}, \mathrm{P}=0.259)$. The two groups did not differ in terms of hospital stay, postoperative eGFR, and change in eGFR.
The rate of positive surgical margin (PSM) was tended to be lower in the MRASE group, but the difference was not statistically significant $(2.2 \%$ vs. $6.3 \%, \mathrm{P}=0.284)$.

The two groups did not differ in terms of the rate of intraoperative complications $(2.2 \%$ vs. $6.3 \%, \mathrm{P}=0.284)$. In the MRASE group the complications included only renal hemorrhage requiring transfusion. In the RAPN group, there were three cases of hemorrhage from the tumor bed requiring transfusion and one case of renal vein injury requiring sutures. The incidence of postoperative complications was also similar ( $10.1 \%$ vs. $14.1 \%, \mathrm{P}=0.405)$, and Clavien-Dindo grade 1 or 2 complications were the majority. Clavien-Dindo grade 3 complications occurred in three cases. One superselective arterial embolization and one ureteral stenting for urinary fistula occurred in RAPN group, and 1 superselective arterial embolization in MRASE group. The median follow-up was 32 months (range, 15-47 months) with MRASE and 30 months (range, 14-45 months) with RAPN. The two groups did not differ for the rate of local recurrence or distant metastases (2.2\% vs. 1.6\%, $\mathrm{P}=1.000)$.

Trifecta was accomplished in $61.2 \%$ and $42.2 \%$ of MRASE and RAPN cases $(\mathrm{P}=0.012)$. On univariable analysis, larger tumor size, higher RENAL score, longer operative time, longer WIT, higher blood loss, longer hospital stay and increased eGFR percentage change were negative predictors for Trifecta achievement (Table 2). On multivariable analysis, procedure type, RENAL score, operative time, and estimated blood loss were positive factors for achievement of Trifecta (Table 3).

\section{Discussion}

The da Vinci Surgical System has been more and more widely used for the treatment of renal tumors (22), and the indications for robotic approach have gradually expanded to more complex and challenging cases (23). The use of the term Trifecta to describe the effectiveness and outcomes of $\mathrm{PN}$ is relatively new and may contribute standardized evaluation of outcomes (24). Trifecta criteria principally consist of absence of complications, negative surgical margins, and minimal renal function decrease. To our knowledge, this is the first analysis to compare RASE with RAPN for T1b renal tumors in the era of Trifecta achievement.

Trifecta assessment for evaluating perioperative outcomes has some limitations due to the lack of a standardized definition. Buffi et al. described the term MIC (negative surgical margin, WIT $<20 \mathrm{~min}$ and no 
Table 1 Clinical characteristics and perioperative data of MRASE and RAPN

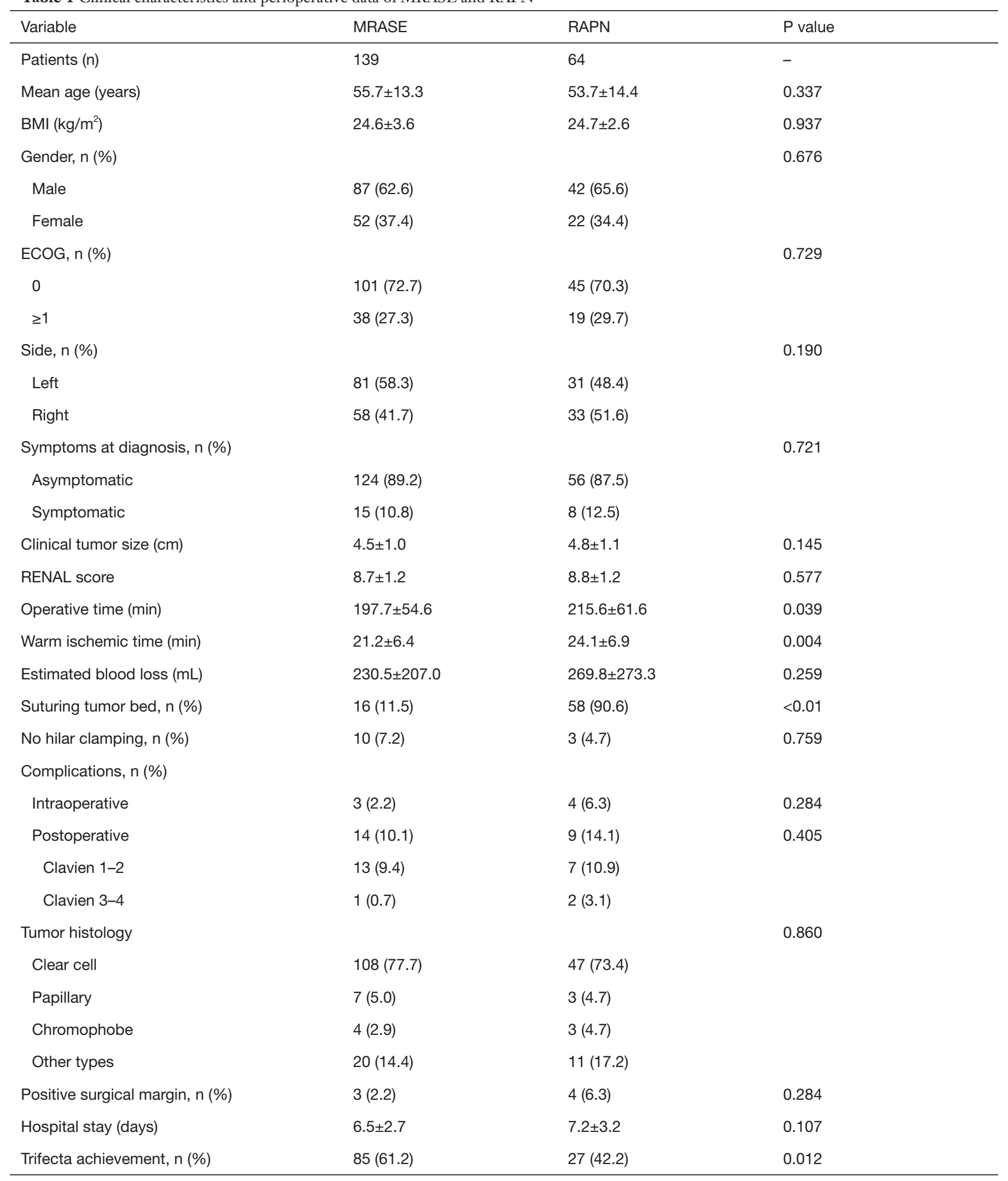

Table 1 (continued) 
Table 1 (continued)

\begin{tabular}{llll}
\hline Variable & MRASE & RAPN & P value \\
\hline Preoperative eGFR $\left(\mathrm{mL} / \mathrm{min} / 1.73 \mathrm{~m}^{2}\right)$ & $100.1 \pm 17.9$ & $101.9 \pm 19.9$ & 0.503 \\
Last eGFR $\left(\mathrm{mL} / \mathrm{min} / 1.73 \mathrm{~m}^{2}\right)$ & $87.7 \pm 18.4$ & $83.3 \pm 17.8$ & 0.111 \\
Change in eGFR $(\%)$ & $-14.0 \pm 18.7$ & $-15.0 \pm 16.1$ & 0.733 \\
\hline
\end{tabular}

MRASE, modified robot-assisted simple enucleation; RAPN, robot-assisted partial nephrectomy; BMI, body mass index; ECOG, Eastern Cooperative Oncology Group; eGFR, estimated glomerular filtration rate.

Table 2 Clinical characteristics and perioperative data of Trifecta achievement

\begin{tabular}{|c|c|c|c|}
\hline Variable & Trifecta & No Trifecta & $P$ value \\
\hline Mean age (years) & $55.1 \pm 14.3$ & $55.0 \pm 12.8$ & 0.964 \\
\hline BMI $\left(\mathrm{kg} / \mathrm{m}^{2}\right)$ & $24.3 \pm 2.9$ & $25.0 \pm 3.7$ & 0.116 \\
\hline ECOG, n (\%) & & & 0.442 \\
\hline$\geq 1$ & $29(25.9)$ & $28(30.8)$ & \\
\hline Side, $\mathrm{n}(\%)$ & & & 0.233 \\
\hline Left & $66(58.9)$ & $46(50.5)$ & \\
\hline Right & $46(41.1)$ & $45(49.5)$ & \\
\hline RAPN & $27(24.1)$ & $37(40.7)$ & \\
\hline Clinical tumor size $(\mathrm{cm})$ & $4.3 \pm 1.0$ & $4.9 \pm 0.9$ & $<0.01$ \\
\hline RENAL score & $8.4 \pm 1.2$ & $9.0 \pm 1.2$ & $<0.01$ \\
\hline Operative time (min) & $176.6 \pm 40.4$ & $236.3 \pm 58.2$ & $<0.01$ \\
\hline Warm ischemic time (min) & $18.0 \pm 4.8$ & $27.2 \pm 4.9$ & $<0.01$ \\
\hline Estimated blood loss (mL) & $167.4 \pm 138.5$ & $335.8 \pm 281.5$ & $<0.01$ \\
\hline Hospital stay (days) & $6.3 \pm 2.5$ & $7.3 \pm 3.3$ & 0.016 \\
\hline Preoperative eGFR $\left(\mathrm{mL} / \mathrm{min} / 1.73 \mathrm{~m}^{2}\right)$ & $100.4 \pm 18.1$ & $100.8 \pm 19.2$ & 0.689 \\
\hline
\end{tabular}

BMI, body mass index; ECOG, Eastern Cooperative Oncology Group; MRASE, modified robot-assisted simple enucleation; RAPN, robotassisted partial nephrectomy; eGFR, estimated glomerular filtration rate.

major complications) as their ideal outcome definition (25). Khalifeh et al. summarized Trifecta as negative surgical margins, no perioperative complications and a WIT $\leq 25 \mathrm{~min}$ (26). Hung et al. defined Trifecta as a composite of negative margins, no urological complications and an actual $v s$. predicted postoperative eGFR $>90 \%$ (24). The need of achieving negative margins and no complications is common among all these definitions, but there is debate as to whether the WIT or renal function preservation determined by the eGFR, should be used as a criterion 
Table 3 Univariable and multivariable analysis for Trifecta achievement

\begin{tabular}{|c|c|c|c|c|c|c|}
\hline Variable & \multicolumn{3}{|c|}{ Univariable analysis } & \multicolumn{3}{|c|}{ Multivariable analysis } \\
\hline Age & 1.000 & $0.980-1.021$ & 0.964 & 0.979 & $0.952-1.006$ & 0.129 \\
\hline BMI & 0.934 & $0.857-1.017$ & 0.118 & 1.005 & $0.899-1.124$ & 0.924 \\
\hline RAPN vs. MRASE & 2.155 & $1.182-3.937$ & 0.012 & 2.302 & $1.039-5.100$ & 0.040 \\
\hline RENAL score & 0.657 & $0.514-0.841$ & $<0.01$ & 0.702 & $0.510-0.966$ & 0.030 \\
\hline Preoperative eGFR & 0.987 & $0.974-1.031$ & 0.689 & 1.002 & $0.983-1.022$ & 0.819 \\
\hline Operative time & 0.961 & $0.951-0.972$ & $<0.01$ & 0.969 & $0.957-0.980$ & $<0.01$ \\
\hline Estimated blood loss & 0.995 & $0.993-0.997$ & $<0.01$ & 0.997 & $0.995-1.000$ & 0.024 \\
\hline
\end{tabular}

OR, odds ratio; $\mathrm{Cl}$, confidence interval; RAPN, robot-assisted partial nephrectomy; MRASE, modified robot-assisted simple enucleation;

$\mathrm{BMI}$, body mass index; eGFR, estimated glomerular filtration rate.

for the Trifecta definition. The WIT is an important indicator for evaluating immediate renal function, and a WIT between 20 and $30 \mathrm{~min}$ is safe (24). Because WIT of $25 \mathrm{~min}$ is reported to be significantly associated with newly developed stage IV chronic kidney disease, it was set as the cutoff in this series (27).

In this study, the MRASE group showed shorter operative time and WIT than RAPN. The estimated blood loss was comparable in both groups. Tumor bed suturing was performed with a lower frequency in the MRASE group than RAPN. The incidence of complications and PSM was comparable. The type of procedure, RENAL score, operative time, and blood loss were positive factors for Trifecta achievement.

A high tumor complexity score was a predictor of no Trifecta achievement. Several previous studies have reported on the correlation between a high tumor complexity scores, and a longer WIT and a higher incidence of complications (28). The estimated blood loss and operative time are indicators for the difficulty of operation and are likely to be associated with developing perioperative complications (29).

The technique of tumor enucleation consists of removing the tumor by blunt dissection along the natural plane (30-32). In the present study, despite the two groups showed similar patient demographics and tumor characteristics, the MRASE group had a less frequency of tumor bed suturing and accomplishment of delayed vascular control, which may help shorten the WIT. These results may be explained by the effects of tumor compression and some pathologic changes like glomerulosclerosis and vascular degeneration close to the tumor (33). Some previous studies also showed superior surgical outcomes of SE compared with standard PN (8,34). Moreover, the MRASE technique reduced damaging the residual renal tissue, which would benefit the renal function, and reduce severe bleeding and urinary collecting system laceration.

One of the challenges in SE is the achievement of negative surgical margins. Several studies have shown doubt about the PSM risk and local recurrence due to the surgical excision method. However, the tumor pseudocapsule and the degenerated adjacent parenchyma could prevent the tumor invasion into the normal renal parenchyma (35). During MRASE, the tumor capsule acts as the surgical landmark, and surgeons can use this natural plane for tumor enucleation. Moreover, the 3D surgical view and fully flexible instrument of robotic system can help avoid the risk of PSM. In the present study, the incidence of PSM in MRASE group was only $2.2 \%$. During standard PN, because no anatomical landmarks can be used, it is difficult to determine the excision plane and easy to enter into the tumor especially in endogenic cases.

There are limitations to the present study. First, this is a retrospective study. Second, this study lacks of randomization and a standardized manner of the procedure choice. Third, the choice of surgical technique was on the basis of tumor characteristics and surgeon's preference, which lead to selection bias. Moreover, the decrease in eGFR of the operated kidney could not be estimated, and a volumetric study was not included. 


\section{Conclusions}

MRASE was superior to RAPN for perioperative surgical results measured by Trifecta in T1b renal tumors. Moreover, MRASE has better outcomes for shorter operative time, shorter WIT, and less need for tumor bed suturing as compared with RAPN. The type of procedure, RENAL score, estimated blood loss, and operative time were predictive factors for the Trifecta achievement.

\section{Acknowledgments}

Funding: This work was supported by the National Natural Science Foundation of China (81772710 and 81572519).

\section{Footnote}

Reporting Checklist: The authors have completed the STROBE reporting checklist. Available at http://dx.doi. org/10.21037/tau-20-1153

Data Sharing Statement: Available at http://dx.doi. org/10.21037/tau-20-1153

Conflicts of Interest: All authors have completed the ICMJE uniform disclosure form (available at http://dx.doi. org/10.21037/tau-20-1153). The authors have no conflicts of interest to declare.

Ethical Statement: The authors are accountable for all aspects of the work in ensuring that questions related to the accuracy or integrity of any part of the work are appropriately investigated and resolved. The study was conducted in accordance with the Declaration of Helsinki (as revised in 2013). The study was approved by institutional ethics committee of Nanjing Drum Tower Hospital (2019153-02) and informed consent was taken from all the patients.

Open Access Statement: This is an Open Access article distributed in accordance with the Creative Commons Attribution-NonCommercial-NoDerivs 4.0 International License (CC BY-NC-ND 4.0), which permits the noncommercial replication and distribution of the article with the strict proviso that no changes or edits are made and the original work is properly cited (including links to both the formal publication through the relevant DOI and the license). See: https://creativecommons.org/licenses/by-nc-nd/4.0/.

\section{References}

1. Ljungberg B, Bensalah K, Canfield S, et al. EAU guidelines on renal cell carcinoma: 2014 update. Eur Urol 2015;67:913-24.

2. Bhatt JR, Finelli A. Landmarks in the diagnosis and treatment of renal cell carcinoma. Nat Rev Urol 2014;11:517-25.

3. Li QL, Guan HW, Zhang QP, et al. Optimal margin in nephron-sparing surgery for renal cell carcinoma $4 \mathrm{~cm}$ or less. Eur Urol 2003;44:448-51.

4. Ljungberg B, Hanbury DC, Kuczyk MA, et al. Renal cell carcinoma guideline. Eur Urol 2007;51:1502-10.

5. Antonelli A, Cozzoli A, Nicolai M, et al. Nephron-sparing surgery versus radical nephrectomy in the treatment of intracapsular renal cell carcinoma up to $7 \mathrm{~cm}$. Eur Urol 2008;53:803-9.

6. Castilla EA, Liou LS, Abrahams NA, et al. Prognostic importance of resection margin width after nephronsparing surgery for renal cell carcinoma. Urology 2002;60:993-7.

7. Satkunasivam R, Tsai S, Syan S, et al. Robotic unclamped "minimal-margin" partial nephrectomy: ongoing refinement of the anatomic zero-ischemia concept. Eur Urol 2015;68:705-12.

8. Lu Q, Zhao X, Ji C, et al. Modified laparoscopic simple enucleation with single-layer suture technique versus standard laparoscopic partial nephrectomy for treating localized renal cell carcinoma. Int Urol Nephrol 2017;49:239-45.

9. Minervini A, Campi R, Sessa F, et al. Positive surgical margins and local recurrence after simple enucleation and standard partial nephrectomy for malignant renal tumors: systematic review of the literature and meta-analysis of prevalence. Minerva Urol Nefrol 2017;69:523-38.

10. Gettman MT, Blute ML, Chow GK, et al. Robotic-assisted laparoscopic partial nephrectomy: technique and initial clinical experience with DaVinci robotic system. Urology 2004;64:914-8.

11. Wang Y, Ma X, Huang Q, et al. Comparison of robotassisted and laparoscopic partial nephrectomy for complex renal tumours with a RENAL nephrometry score $\geq 7$ : peri-operative and oncological outcomes. BJU Int 2016;117:126-30.

12. Minervini A, Vittori G, Antonelli A, et al. Open versus robotic-assisted partial nephrectomy: a multicenter comparison study of perioperative results and complications. World J Urol 2014;32:287-93. 
13. Mottrie A, De Naeyer G, Schatteman P, et al. Impact of the learning curve on perioperative outcomes in patients who underwent robotic partial nephrectomy for parenchymal renal tumours. Eur Urol 2010;58:127-32.

14. Zhao X, Lu Q, Campi R, et al. Endoscopic Robotassisted Simple Enucleation Versus Laparoscopic Simple Enucleation With Single-layer Renorrhaphy in Localized Renal Tumors: A Propensity Score-matched Analysis From a High-volume Centre. Urology 2018;121:97-103.

15. Oken MM, Creech RH, Tormey DC, et al. Toxicity and response criteria of the Eastern Cooperative Oncology Group. Am J Clin Oncol 1982;5:649-55.

16. Patard JJ, Leray E, Cindolo L, et al. Multi-institutional validation of a symptom based classification for renal cell carcinoma. J Urol 2004;172:858-62.

17. Kutikov A, Uzzo RG. The R.E.N.A.L. nephrometry score: a comprehensive standardized system for quantitating renal tumor size, location and depth. J Urol 2009;182:844-53.

18. Dindo D, Demartines N, Clavien PA. Classification of surgical complications: a new proposal with evaluation in a cohort of 6336 patients and results of a survey. Ann Surg 2004;240:205-13.

19. Levey AS, Bosch JP, Lewis JB, et al. A more accurate method to estimate glomerular filtration rate from serum creatinine: a new prediction equation. Modification of Diet in Renal Disease Study Group. Ann Intern Med 1999;130:461-70.

20. Khalifeh A, Autorino R, Hillyer SP, et al. Comparative outcomes and assessment of trifecta in 500 robotic and laparoscopic partial nephrectomy cases: a single surgeon experience. J Urol 2013;189:1236-42.

21. Rogers CG, Singh A, Blatt AM, et al. Robotic partial nephrectomy for complex renal tumors: surgical technique. Eur Urol 2008;53:514-21.

22. Choi JE, You JH, Kim DK, et al. Comparison of perioperative outcomes between robotic and laparoscopic partial nephrectomy: a systematic review and metaanalysis. Eur Urol 2015;67:891-901.

23. Long JA, Yakoubi R, Lee B, et al. Robotic versus laparoscopic partial nephrectomy for complex tumors: comparison of perioperative outcomes. Eur Urol 2012;61:1257-62.

24. Hung AJ, Cai J, Simmons MN, et al. "Trifecta" in partial nephrectomy. J Urol 2013;189:36-42.

25. Buffi N, Lista G, Larcher A, et al. Margin, ischemia, and complications (MIC) score in partial nephrectomy: a new system for evaluating achievement of optimal outcomes in nephron-sparing surgery. Eur Urol 2012;62:617-8.

26. Khalifeh A, Autorino R, Eyraud R, et al. Three-year oncologic and renal functional outcomes after robotassisted partial nephrectomy. Eur Urol 2013;64:744-50.

27. Mir MC, Ercole C, Takagi T, et al. Decline in renal function after partial nephrectomy: etiology and prevention. J Urol 2015;193:1889-98.

28. Ficarra V, Bhayani S, Porter J, et al. Predictors of warm ischemia time and perioperative complications in a multicenter, international series of robot-assisted partial nephrectomy. Eur Urol 2012;61:395-402.

29. Mathieu R, Verhoest G, Droupy S, et al. Predictive factors of complications after robot-assisted laparoscopic partial nephrectomy: a retrospective multicentre study. BJU Int 2013;112:E283-9.

30. Puppo P, Introini C, Calvi P, et al. Long term results of excision of small renal cancer surrounded by a minimal layer of grossly normal parenchyma: review of 94 cases. Eur Urol 2004;46:477-81.

31. Jeong SJ, Kim KT, Chung MS, et al. The prognostic value of the width of the surgical margin in the enucleoresection of small renal cell carcinoma: an intermediate-term followup. Urology 2010;76:587-92.

32. Minervini A, Serni S, Tuccio A, et al. Simple enucleation versus radical nephrectomy in the treatment of $\mathrm{pT} 1 \mathrm{a}$ and p T1b renal cell carcinoma. Ann Surg Oncol 2012;19:694-700.

33. Azhar RA, de Castro Abreu AL, Broxham E, et al. Histological analysis of the kidney tumor-parenchyma interface. J Urol 2015;193:415-22.

34. Longo N, Minervini A, Antonelli A, et al. Simple enucleation versus standard partial nephrectomy for clinical T1 renal masses: perioperative outcomes based on a matched-pair comparison of 396 patients (RECORd project). Eur J Surg Oncol 2014;40:762-8.

35. Minervini A, di Cristofano C, Lapini A, et al. Histopathologic analysis of peritumoral pseudocapsule and surgical margin status after tumor enucleation for renal cell carcinoma. Eur Urol 2009;55:1410-8.

Cite this article as: Zhao X, Lu Q, Ji X, Liu G, Qiu X, Zhang S, Li X, Zhang G, Guo H. Trifecta outcomes of modified robotassisted simple enucleation and standard robot-assisted partial nephrectomy for treating clinical T1b renal cell carcinoma. Transl Androl Urol 2021;10(3):1080-1087. doi: 10.21037/tau-201153 